\title{
The Design and Implementation of Displacement
}

\section{Monitoring System for Tailings Dam}

\author{
Guoshao Chen \\ School of Computer Science and Engineering \\ Xi'an Technological University \\ Xi'an, 710021, China \\ e-mail: $1825247141 @ q q . c o m$
}

\author{
Zhichao Lian \\ School of Computer Science and Engineering, \\ Xi'an Technological University, \\ Xi'an, 710021, China \\ e-mail: 965941167@qq.com
}

Abstract-Non-coal mining generally produces a large amount of tailings, and tailings usually accumulate in the canyon between two mountains, and the downstream of the tailings is intercepted by the dam. The dam is called a tailings dam. The development of safety monitoring technology for tailings dam is accompanied by the history of dam construction. The safety of tailings dam is not only related to the normal operation of mining industry, but also to the life safety of downstream residents. Once the tailings dam break, the loss caused by the dam is inestimable. In this paper, the laser collimation system is used to monitor the displacement of the dam, and the center of light is measured by the center of gravity, and the laser beam is reconstructed by a lens. The method has the advantages of high accuracy and simultaneous detection of horizontal displacement and vertical displacement of tailings dam. It is a new monitoring technology for dam displacement and has good application prospects.

Keywords-Dam Displacement; Center of Gravity Method; Laser Spot

\section{SUMMARY}

Human beings have begun to develop and use mineral resources a long time ago. China has a rich variety of mining industries, and the mining industry is the basis of various industries. Minerals can't be $100 \%$ purity. During the smelting process, the impurities in ores are separated. Because the value of impurities can be very small, they accumulate in tailings. With the improvement of smelting technology, the utilization ratio of minerals is becoming higher and higher, but there are still some tailings. A tailings dam has been formed and the tailings dam is used to prevent the dams from tailings. In our country, thousands of such tailing dams are standing large and small. The collapse of the tailings dam occurred, which caused great loss to the life and property of the country and the people, and caused serious pollution to the natural environment. In September 8, 1998, a special dam break occurred in Linfen, Shanxi, which killed 254 people and had a very bad impact on the society. In September 21, 2010, tin tailing dam of Guangdong Xinyi Qian Pai Village in Zijin Mining Co. the dam failure occurred, the accident caused 6 people missing, 5 people were killed and 7 injured.

Because of various reasons, many countries have occurred dam break in history. In December 2, 1959, the French Marce broke down. Because the management failed to analyze the dam monitoring data in time, it resulted in a disaster that could be avoided. Although the history of damming is accompanied by the history of human civilization, it has a history of thousands of years. However, due to various uncertain factors of the dam body, it is difficult to detect the safety of the dam. Early monitoring is simply a simple appearance check, and no scientific monitoring means. By the middle of twentieth Century, the dam safety monitoring theory system was basically formed. With the appearance of some monitoring instruments and monitoring methods, vertical line method, triangulation method and precise leveling method were used to measure horizontal displacement and vertical displacement. 
According to the technical code for safety monitoring of dam (SL 60-94) promulgated in 1994 and the technical code for safety monitoring of concrete dams issued in 2003 (DL/T5178-2003), dam safety monitoring projects generally include the following 5 aspects.

1) Monitoring of working conditions: also called environmental monitoring, mainly including water level, water temperature and freezing and so on.

2) Deformation monitoring: mainly including the horizontal displacement of the dam and the vertical displacement of the dam.
3) Water leakage monitoring: mainly including the infiltration line of the dam.

4) Pressure monitoring: mainly including the temperature of the dam, the earth pressure and so on.

5) Other monitoring: mainly including earthquake monitoring.

\section{SYSTEM OVERALL DESIGN}

In this paper, the image processing method is used to measure the displacement of the dam, and its structure is shown in Figure 1

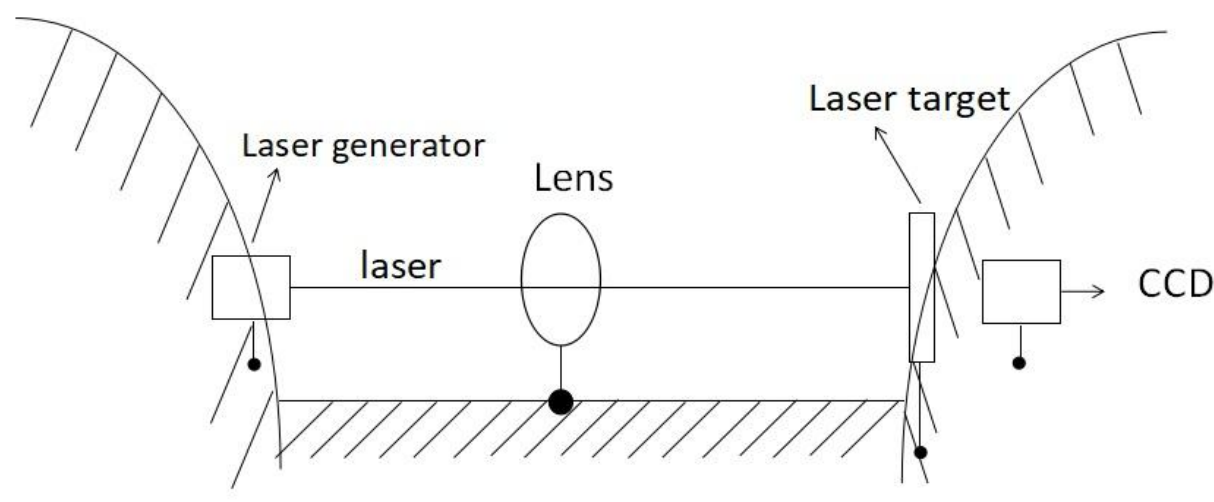

Figure 1. System structure

The laser and laser receiver are fixed on both sides of the mountain. The lens is mounted on the dam body. The laser uses a helium neon laser, and the laser emitted from the light source is projected to the laser receiver through the lens. The laser will form a spot on the receiver. The laser, lens, and laser receiver are in the same line in the ideal condition. When the dam displacement occurs, it will drive the lens to move. The corresponding image is also moving on the laser receiver. By measuring the displacement of the spot on the detector, we can get the displacement of lens system further.

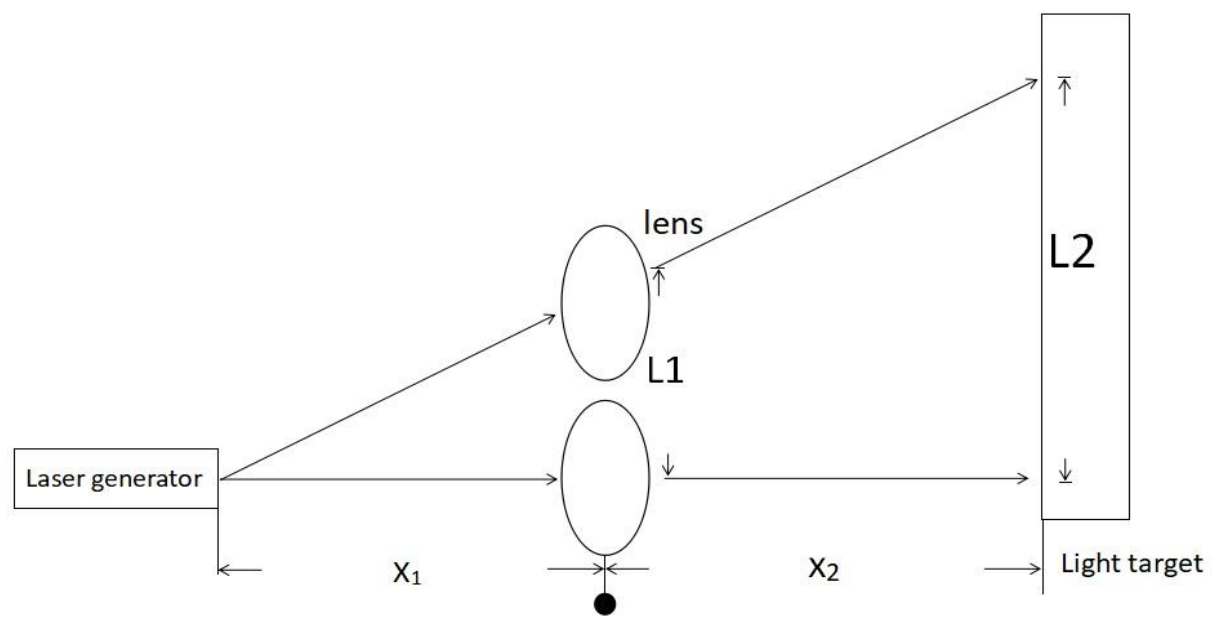

Figure 2. Monitoring schematic 
Figure 2 is a monitoring schematic. $\mathrm{L}$ is a laser, a lens is $\mathrm{M}$, and the center of $\mathrm{M}$ is $\mathrm{O}$ and $\mathrm{O}^{\prime}$, $\mathrm{y}$ and $\mathrm{y}^{\prime}$ as a laser spot on the laser receiver. When the lens is at $\mathrm{O}$, the $\mathrm{L}$ passes through the $\mathrm{M}$ imaging at the $\mathrm{Y}$ point; when the lens is moved to $\mathrm{O}$ ', the spot moves to $\mathrm{y}^{\prime}$. The displacement distance of the lens can be calculated by the CCD system, which is behind the laser receiver, to collect the displacement of the spot. According to the trigonometric relationship

$$
\frac{x_{1}}{\left(x_{1}+x_{2}\right)}=\frac{l_{1}}{l_{2}}
$$

Assuming that the location of the lens is only moving up and down, the length of $\mathrm{x} 1$ and $\mathrm{x} 2$ is known. As long as we can detect the displacement of Y accurately, we can further calculate the displacement of the dam. The deformation of the dam body can be obtained by measuring the displacement of each measuring point installed on the dam body.

The images captured by the CCD camera should be a perfect dot in the ideal situation. But in practice, there are inevitably some diffraction phenomena, and all kinds of other disturbances will be the deformation of the spot, so that the ideal center position of the spot will be deviate from the actual position. So we must first filter the image collected by CCD to carry out the next process.

\section{GRAVITY METHOD}

In this paper, the center of laser spot is extracted by the method of center of gravity extraction. When using the center of gravity method to deal with the spot, the image is first two valued, and then the spot area is identified. The accuracy of this method is relatively high and the speed is faster. The center of gravity method is only applicable to the symmetry pattern. If the image is asymmetrical, there will be a lot of deviation.

The basic principle of the gravity method is to replace the geometric center of the image with the energy center of the image. The gray value of the pixel of the image is $\mathrm{P}$. The center of gravity coordinates are $(x, y)$

$$
\begin{aligned}
& x=\frac{\sum x_{i} \times p_{i}}{\sum p_{i}} \\
& y=\frac{\sum y_{i} \times p_{i}}{\sum p_{i}}
\end{aligned}
$$

$i=1,2,3 \ldots . n$

In order to improve the reliability of the system, every measuring point must be measured several times, and the position of the spot can be calculated according to the measured data. CCD is repeatedly sampled to get the average value, so as to improve the accuracy.

\section{HARDWARE INTRODUCTION}

The system consists of six parts is shown in figure 3 :

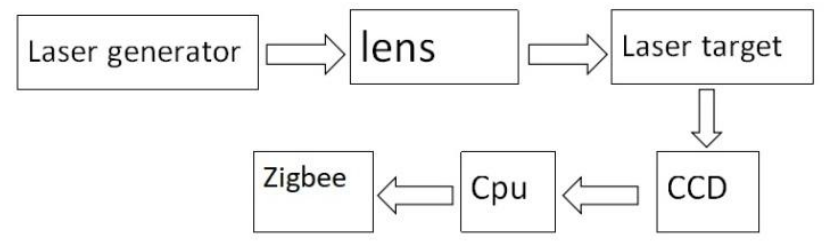

Figure 3. System hardware

\section{A. Laser}

The laser has high luminance, very small divergence angle and good monochromatic property; because of these unique properties, it is used as a quasi straight line in this system.

\section{B. Lens system}

In this system, the beam of laser is reshaped, the quality of beam is improved, and the accuracy of measurement is improved. Optical lens is used to realize it. In practical application, Fresnel Lens is used.

\section{Laser receiver}

The translucent scattering glass similar to the wool glass is convenient for the camera to collect the spot of the light spot. 


\section{Camera}

The receiver module uses the camera, and the application of the camera is very common. In this system, it is mainly used to accept the laser spot image.

\section{E. Processor}

An intelligent chip is used to analyze the image, and the amount of displacement is calculated. It mainly includes hardware design and software design. And the data will be sent to the wireless module.

\section{F. Zigbee wireless module}

The system uses the wireless module to transmit the deformable data to the base station, and overcomes the difficulty of long distance wiring, and uses the popular Zigbee to implement it. Zigbee is a wireless network transmission technology which has been developed in recent years, which is adaptable to short distance, low rate and low power consumption.

\section{CONCLUSION}

In this paper, the dam displacement monitoring system is designed in detail, including hardware structure design and image processing algorithm. The system is tested in the tailings dam, the data is accurate. Due to the transmission of laser in the air, the visibility of the air is reduced, the brightness of the laser spot is insufficient, so the data cannot be collected sometimes.

\section{ACKNOWLEDGMENT}

Fund support: Shaanxi Education Department Special Fund Project number: Shaanxi Education Finance[2013] 23

\section{REFERENCES}

[1] Tailings beach length monitoring system research based on digital image processing Zhang Yulei

[2] Tailings dam displacement monitoring system based on laser collimation method design Ru Naiyu

[3] Tailings safety monitoring system based on Silverlight Zhang Cheng

[4] High precision positioning algorithm for laser spot center research Chen he; Yang Zhihao; Guo pan; Zhang Yinchao; Chen Siying Beijing Institute of Technology Journal.

[5] Astronomical instruments in image tracking algorithm of the comparative study of Wang Lili; Hu Chinese; season Hangxin astronomy. 